\title{
Could JAG1 protein inhibition prevent colorectal cancer?
}

\author{
Erika López-Arribillaga ${ }^{\ddagger}, 1$, Verónica Rodilla ${ }^{\ddagger}, 2$ \& Lluís Espinosa*,3 \\ ${ }^{1}$ Institute for Research in Biomedicine (IRB Barcelona), The Barcelona Institute of Science \& Technology (BIST), Barcelona, Spain \\ ${ }^{2}$ Preclinical Research Program, Vall d'Hebron Institute of Oncology, CIBERONC, Barcelona, Spain \\ ${ }^{3}$ Cancer Research Program, Institut Mar d'Investigacions Mèdiques, CIBERONC, Hospital del Mar, Doctor Aiguader 88, Barcelona \\ 08003, Spain \\ *Author for correspondence: lespinosa@imim.es \\ ${ }^{\ddagger}$ Authors contributed equally
}

"using anti-JAG1 blocking antibodies to specifically target colorectal cancer"

First draft submitted: 4 October 2018; Accepted for publication: 18 October 2018; Published online:

8 January 2019

Keywords: colorectal cancer $\bullet$ Jag1 $\bullet$ Notch $\bullet$ personalized medicine $\bullet$ therapeutic antibodies

Surgery is the primary choice of treatment for nonmetastatic colorectal cancer (CRC) patients in combination with adjuvant therapies, comprising classical chemotherapy and/or radiotherapy. Aberrant Notch activity is a hallmark of a high percentage of colorectal tumors, but a systemic Notch inhibition is highly toxic, therefore not an option. In this Editorial, we discuss the possibility of using anti-JAG1 blocking antibodies to specifically target colorectal cancer cells and avoid the intestinal toxicity of Notch blockade.

\section{Jag1}

Notch family is formed by four different receptors (Notch1-4), three Delta ligands (Dll1, 2 and 4) and two Jagged ligands (Jag1 and 2). The ligand binding to the receptor and its subsequent activation is tightly regulated by Pofut 1 and Fringe glycosyl-transferases (Mfng, Lfng and Rfng) [1]. When Notch receptors are glycosylated by Mfng and Lfng, they reduce their affinity for Jagged and become more responsive to Delta-like ligands instead.

Notch signaling is activated by cell-cell contact and has been involved in several physiological processes including stem cell maintenance in various tissues including the intestine [2,3]. $\gamma$-secretase is an essential enzyme for Notch activation - it cleaves the intracellular domain of Notch receptors, which then goes into the nucleus to activate transcription [4]. Moreover, $\gamma$-secretases, concretely ADAM17, can also cleave Jag1 releasing an extracellular soluble form implicated in paracrine signaling between endothelial cells and tumor cells [5].

Based on knockout studies in mice, the main physiological function of Jag1 is regulating vasculogenesis and bile duct formation during embryonic stage [6], whereas it is totally dispensable for the maintenance of intestinal homeostasis [2]. Interestingly, mutations in $J A G 1$ result in Alagille syndrome in humans, an inherited multi-organ developmental disorder [7].

\section{Notch in CRC}

Notch activity is important for different oncogenic processes including colorectal cancer, and it is involved in tumor initiation [8], tumor progression and metastasis [9-11]. While Notch mutations are at the base of its activation in T-cell acute leukemia, activation of Notch in a subset of colorectal tumors is supported by Jag1 overexpression, as a consequence of aberrant $\mathrm{Wnt} / \beta$-catenin activity. This protumorigenic axis involving Wnt-Jag1-Notch1 was previously demonstrated by our group using the $A p c^{\mathrm{Min} /+}$ model crossed to the heterozygous knockout of Jag1 [12]. Although this model did not allow discerning the specific contribution of epithelial Jag1 from the ligand expressed by other cell types, our most recent work has demonstrated that Jag1 deletion in the intestinal epithelial cells prevents $A p c^{\mathrm{Min} /+}$ tumor formation, reduces expression of several intestinal stem cell (ISC) markers, and inhibits tumor spheroid growth and re-passaging, which is a measure of tumor-initiating cell (TIC) activity [13]. This is of 
paramount importance when exploring treatment options, as TICs, which share characteristics with normal stem cells, have been regarded as responsible for tumor relapse, metastasis and resistance to chemotherapy.

\section{Therapies against Notch signaling for treating CRC}

The use of $\gamma$-secretase inhibitors (GSIs) as anti-cancer therapy has been extensively investigated. Preclinical research has consistently shown that GSI treatment inhibits tumorigenesis through different mechanisms, which include reducing angiogenesis and promoting differentiation of cancer cells into the secretory lineage [14,15]. However, GSIassociated toxicity, mainly caused by goblet cell metaplasia and intestinal stem cell loss, is still a major drawback that needs further investigation, although it can be reduced by intermittent dosing and ameliorated by glucocorticoid co-treatment [16]. Still, $\gamma$-secretase activity is not exclusively acting on Notch receptors, and can affect processing of other proteins, which would result in additional undesired side effects [15].

In an attempt to improve treatment efficacy, several monoclonal antibodies selectively targeting Notch 1-3 have already been generated and are currently being tested for clinical applications [17]. Although they seem to reduce toxicity compared with GSIs, they still have some side effects due to the critical role of Notch signaling in ISCs, where Notch1 and 2 knockout was shown to exhaust the stem cell compartment [18]. The previously mentioned demonstration that Jag1 is dispensable for intestinal homeostasis open the possibility of targeting Jag1 in human colorectal cancer patients with less or no intestinal side effects.

\section{Which patients could benefit from JAG1 blocking therapy?}

In our recent work, we have analyzed a subset of 200 CRC patient samples and found a mixed pattern for JAG1 expression with no correlation between JAG1 and ICN1 (Intra-Cellular Notch1, active form of Notch1) levels at advanced carcinoma stages. However, we found that high Notch activity in tumors expressing JAG1 that are MFNG negative is predictive of poor survival. We experimentally validated that reduced MFNG levels determine a distinctive addiction to JAG1 and define a subset of tumors that respond to anti-JAG1 therapy [13]. The possibility of using anti-JAG1 antibodies as an alternative therapy to conventional chemotherapy, which is not specific and gives strong intestinal side effects, should be tested in preclinical trials. Moreover, using the recently established system of patient-derived tumoroids we can pretest the efficacy of this therapy (alone or in combination) in individual patients.

Furthermore, this therapeutic strategy could be applied to reduce adenoma formation in familial adenomatous polyposis (FAP) patients and to treat other tumor types such as breast, cervical and ovarian cancer where JAG1 also exerts pro-oncogenic functions [19]. In contrast, in acute myeloid leukemia, JAG1 has been described as a tumor suppressor gene, which reduces cancer cell growth and its expression correlates with favorable prognosis [20].

In summary, we propose that cancer patients carrying tumors with high levels of ICN1, JAG1 and low levels of MFNG would be strong candidates for anti-JAG1 therapies.

Financial \& competing interests disclosure

We thank Instituto de Salud Carlos III (PIE15/00008 and PI16/00437)/FEDER and Generalitat de Catalunya (2017SGR135) for supporting this work. The authors have no other relevant affiliations or financial involvement with any organization or entity with a financial interest in or financial conflict with the subject matter or materials discussed in the manuscript apart from those disclosed.

No writing assistance was utilized in the production of this manuscript.

\section{References}

1. Kovall RA, Gebelein B, Sprinzak D, Kopan R. The canonical notch signaling pathway: structural and biochemical insights into shape, sugar, and force. Dev. Cell 41, 228-241 (2017).

2. Pellegrinet L, Rodilla V, Liu Z et al. Dll1- and Dll4-mediated notch signaling are required for homeostasis of intestinal stem cells. Gastroenterology 140, 1230-1240 (2011).

3. López-Arribillaga E, Rodilla V, Pellegrinet $\mathrm{L}$ et al. Bmi1 regulates murine intestinal stem cell proliferation and self-renewal downstream of Notch. Development 142, 41-50 (2015).

4. Bigas A, Espinosa L. The multiple usages of Notch signaling in development, cell differentiation and cancer. Curr. Opin. Cell Biol. 55 , $1-7$ (2018).

5. Lu J, Ye X, Fan F et al. Endothelial cells promote the colorectal cancer stem cell phenotype through a soluble form of Jagged-1. Cancer Cell 23, 171-185 (2013). 
6. Xue Y, Gao X, Lindsell CE et al. Embryonic lethality and vascular defects in mice lacking the Notch ligand Jagged1. Hum. Mol. Genet. 8, 723-730 (1999).

7. Oda T, Elkahloun AG, Pike BL et al. Mutations in the human Jagged1 gene are responsible for Alagille syndrome. Nat. Genet. 16, 235-242 (1997).

8. Fre $\mathrm{S}$, Pallavi SK, Huyghe $\mathrm{M}$ et al. Notch and Wnt signals cooperatively control cell proliferation and tumorigenesis in the intestine. Proc. Natl Acad. Sci. USA 106, 6309-6314 (2009).

9. Chanrion M, Kuperstein I, Barrière $\mathrm{C}$ et al. Concomitant Notch activation and $\mathrm{p} 53$ deletion trigger epithelial-to-mesenchymal transition and metastasis in mouse gut. Nat. Commun. 5, 5005 (2014).

10. Sonoshita M, Aoki M, Fuwa $\mathrm{H}$ et al. Suppression of colon cancer metastasis by aes through inhibition of notch signaling. Cancer Cell 19, 125-137 (2011).

11. Sonoshita M, Itatani Y, Kakizaki F et al. Promotion of colorectal cancer invasion and metastasis through activation of NOTCH-DAB1-ABL-RHOGEF protein TRIO. Cancer Discov. 5, 198-211 (2015).

12. Rodilla V, Villanueva A, Obrador-Hevia A et al. Jagged1 is the pathological link between Wnt and Notch pathways in colorectal cancer. Proc. Natl Acad. Sci. USA 106, 6315-6320 (2009).

13. López-Arribillaga E, Rodilla V, Colomer $\mathrm{C}$ et al. Manic Fringe deficiency imposes Jagged 1 addiction to intestinal tumor cells. Nat. Commun. 9, 2992 (2018).

14. van Es JH, van Gijn ME, Riccio O et al. Notch/gamma-secretase inhibition turns proliferative cells in intestinal crypts and adenomas into goblet cells. Nature 435, 959-963 (2005).

15. Purow B. Notch inhibition as a promising new approach to cancer therapy. Adv. Exp. Med. Biol. 727, 305-319 (2012).

16. Real PJ, Tosello V, Palomero T et al. Gamma-secretase inhibitors reverse glucocorticoid resistance in $\mathrm{T}$ cell acute lymphoblastic leukemia. Nat. Med. 15, 50-58 (2009).

17. Wu Y, Cain-Hom C, Choy L et al. Therapeutic antibody targeting of individual Notch receptors. Nature 464, 1052-1057 (2010).

18. Riccio O, van Gijn ME, Bezdek AC et al. Loss of intestinal crypt progenitor cells owing to inactivation of both Notch1 and Notch2 is accompanied by derepression of CDK inhibitors p27Kip1 and p57Kip2. EMBO Rep. 9, 377-383 (2008).

19. Li D, Masiero M, Banham AH, Harris AL. The Notch ligand Jagged1 as a target for anti-tumor therapy. Front. Oncol. 4, 254 (2014).

20. Czemerska M, Pluta A, Szmigielska-Kaplon A et al. Jagged-1: a new promising factor associated with favorable prognosis in patients with acute myeloid leukemia. Leuk. Lymphoma 56, 401-406 (2015). 
